\title{
Assessing the vertical transmission potential of dengue virus in field-reared Aedes aegypti using patient-derived blood meals in $\mathrm{Ho}$ Chi Minh City, Vietnam
}

\author{
Daniela da Silva Goncalves ${ }^{1}$, Kien Duong Thi Hue ${ }^{1}$, Vi Tran Thuy ${ }^{1}$, Nhu Vu Tuyet ${ }^{1}$, Giang Nguyen Thi' \\ Van Huynh Thi Thuy ${ }^{1}$, Trang Huynh Thi Xuan ${ }^{1}$, Dui Le Thi ${ }^{1}$, Long Thi Vo ${ }^{1}$, Huynh Le Anh Huy ${ }^{1}$, \\ Nguyen Thi Van Thuy ${ }^{1}$, Bridget A. Wills ${ }^{1}$, Phong Nguyen Thanh ${ }^{2}$, Cameron P. Simmons ${ }^{1,3}$ \\ and Lauren B. Carrington ${ }^{1 *}$
}

\begin{abstract}
Background: Dengue viruses (DENV) can be transmitted from an adult female Aedes aegypti mosquito through the germ line to the progeny; however, there is uncertainty if this occurs at a frequency that is epidemiologically significant. We measured vertical transmission of DENV from field-reared Ae. aegypti to their F1 progeny after feeding upon blood from dengue patients. We also examined the transmission potential of F1 females.

Methods: We examined the frequency of vertical transmission in field-reared mosquitoes, who fed upon blood from acutely viremic dengue patients, and the capacity for vertically infected females to subsequently transmit virus horizontally, in two sets of experiments: (i) compared vertical transmission frequency of field-reared Ae. aegypti and Ae. albopictus, in individual progeny; and (ii) in pooled progeny derived from field- and laboratory-reared Ae. aegypti.

Results: Of 41 DENV-infected and isofemaled females who laid eggs, only a single female (2.43\%) transmitted virus to one of the F1 progeny, but this F1 female did not have detectable virus in the saliva when 14 days-old. We complemented this initial study by testing for vertical transmission in another 460 field-reared females and $>900$ laboratoryreared counterparts but failed to provide any further evidence of vertical virus transmission.

Conclusions: In summary, these results using field-reared mosquitoes and viremic blood from dengue cases suggest that vertical transmission is uncommon. Field-based studies that build on these observations are needed to better define the contribution of vertical DENV transmission to dengue epidemiology.
\end{abstract}

Keywords: Vertical transmission, Dengue virus (DENV), Mosquitoes, Aedes aegypti, Aedes albopictus

\section{Background}

Dengue is a mosquito-borne viral disease. There are an estimated 390 million infections per year, and 3.9 billion people living in 128 countries at risk of infection [1,2].

\footnotetext{
*Correspondence: Ibcarrington@gmail.com

1 Oxford University Clinical Research Unit, Wellcome Trust Major Overseas Programme, District 5, Ho Chi Minh City, Vietnam

Full list of author information is available at the end of the article
}

Dengue is caused by one of four dengue virus (DENV) serotypes, which are transmitted primarily by Aedes aegypti and Ae. albopictus mosquitoes [3]. In recent years, both species have expanded their geographical distribution and, predictions of various climate change scenarios suggest their distribution will increase further. Such scenarios are likely to trigger emergence and reemergence of arboviruses such as dengue worldwide [4].

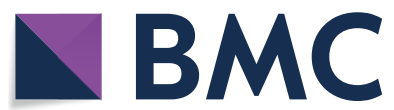

c The Author(s) 2020. This article is licensed under a Creative Commons Attribution 4.0 International License, which permits use, sharing, adaptation, distribution and reproduction in any medium or format, as long as you give appropriate credit to the original author(s) and the source, provide a link to the Creative Commons licence, and indicate if changes were made. The images or other third party material in this article are included in the article's Creative Commons licence, unless indicated otherwise in a credit line to the material. If material is not included in the article's Creative Commons licence and your intended use is not permitted by statutory regulation or exceeds the permitted use, you will need to obtain permission directly from the copyright holder. To view a copy of this licence, visit http://creativeco mmons.org/licenses/by/4.0/. The Creative Commons Public Domain Dedication waiver (http://creativecommons.org/publicdomain/ zero/1.0/) applies to the data made available in this article, unless otherwise stated in a credit line to the data. 
DENV is sustained naturally in a horizontal, humanmosquito transmission cycle. Transmission of virus between humans via the mosquito vector is a by-product of a female mosquito's need for protein to develop the eggs [5]. Vertical transmission (VT) of virus from mother to progeny may be an alternative mechanism for the virus to maintain itself in circulation in nature [6,7]. Should there be transient suboptimal conditions for horizontal transmission (HT), some level of vertical transmission could increase the probability of the pathogen persisting [8]. In the laboratory, vertical transmission can even be observed over consecutive generations in Ae. aegypti [9]. Mosquito rearing temperature $[10,11]$, the strain of mosquitoes and the strain of virus have been suggested to influence vertical transmission $[12,13]$. Despite this, several studies suggest that the frequency of vertical DENV transmission in the field is negligible $[14,15]$ or uncertain $[16,17]$.

While it is impossible to determine whether a virusinfected female caught from the field acquired the virus horizontally or vertically, female infection frequencies in nature can range from $<1 \%$ up to $10 \%$ [18-20]. As neither immatures nor adult males feed on human blood, detection of virus in these individuals, by default, is indicative of vertical transmission from mother to offspring. In the field, estimates of vertical transmission in this group vary from $0 \%[17,19]$ up to around $20 \%$ in pooled larval samples or based on the Ovitraps Index (number of ovitraps with positive eggs divided by the total number of ovitraps installed) $[14,15]$.

To our knowledge, no studies have investigated whether vertically infected females are capable of reestablishing an infection in the natural mosquito-human transmission cycle during their next feeding event. Failing to produce viral loads high enough to transmit DENV to a naïve human host, a vertically infected female may effectively end up a dead-end host.

The objectives of this study were to estimate the proportion of $F_{0}$ females that transmitted virus to their progeny after viremic blood meals from acutely infected dengue patients and the proportion of vertically infected $F_{1}$ females that had infectious saliva. We assessed these vertical transmission indices in two sets of experiments, one testing individual field-reared Ae. aegypti and Ae. albopictus and their individual $\mathrm{F}_{1}$ progeny, after feeding on the viremic blood of 25 acutely infected dengue patients. With an additional five patient-derived blood meals, we compared the prevalence of DENV infection in pools of $\mathrm{F}_{1}$ mosquitoes produced by field-reared $A e$. aegypti, and those reared under laboratory conditions.

\section{Methods}

Patient cohorts and healthy volunteers

Forty patient participants were enrolled between November 2018 and September 2019, with written informed consent obtained by qualified staff from Hospital for Tropical Diseases (HTD). The patients were enrolled on Ward D (male adult dengue ward) of HTD, based on the following inclusion criteria: (i) $\geq 15$ years of age; (ii) an inpatient at HTD with $<96 \mathrm{~h}$ fever at the time of screening; (iii) clinical signs and symptoms consistent with dengue; and (v) written informed consent. Exclusion criteria were: (i) patients who were unconscious or severely ill; and (ii) pregnant women. Only enrolled patients who were NS1 rapid test-positive had venous blood drawn for blood-feeding mosquitoes. An aliquot of each blood sample was taken for quantification of virus and virus serotyping (see Dengue virus diagnostics below), and the remaining was transported to the insectary for blood-feeding.

Healthy volunteers, who also provided written informed consent, provided venous blood samples for experimental feeding (see "Infectious and non-infectious blood meals" below) and for colony maintenance of laboratory-reared mosquitoes. The tympanic temperature of all volunteers was measured prior to blood draws to confirm the healthy volunteers were afebrile.

\section{Experimental overview}

To assess the frequency of VT, we performed two sets of experiments. The first set, using 35 patient-derived blood meals, aimed to compared VT frequency between the two species of field-reared mosquitoes Ae. aegypti and Ae. albopictus, in individual progeny. The second set, using only 5 blood meals, examined whether VT frequencies in Ae. aegypti are influenced by rearing conditions (field-reared $v s$ laboratory-reared), in a slightly modified protocol, with testing for the presence of virus in pooled progeny.

All experiments began with viremic blood meals administered to adult mosquitoes to first infect them with DENV; experimental groups were always exposed in parallel. A second healthy blood meal was later offered to promote egg laying in those same mosquitoes. The first set of experiments administered this non-infectious blood meal on Day 14; the second set administered the blood meal 4 days earlier, on Day 10. All $\mathrm{F}_{1}$ eggs were collected and hatched in batches, according to each $\mathrm{F}_{0}$ female, while the whole body of each mother $\left(\mathrm{F}_{0}\right)$ was collected and tested for DENV infection. After $F_{1}$ mosquito emergence, individuals were harvested for DENV 
screening (see "Hatching and harvesting $\mathrm{F}_{1}$ mosquitoes" below). Screening of mosquitoes in the second set of experiments was performed in pools of up to 8 progeny, due to the large number of $F_{1}$ individuals obtained.

\section{Origin of mosquitoes}

Field-reared $\left(\mathrm{F}_{0}\right)$ immature Ae. aegypti and Ae. albopictus mosquitoes were collected on a weekly basis in households within District 8, Ho Chi Minh City (HCMC), Vietnam. Only fourth-instar larvae or pupae were included in the experiment, to maximise their development time under field conditions. Where colony mosquitoes were used, they were reared under laboratory conditions, and were of the same HCMC genetic background. Laboratory-reared mosquitoes were from generations 45 and 46 , but were outcrossed with $10 \%$ wild-type males every second generation to avoid inbreeding depression, and to maintain genetic variation in the population [20].

\section{Mosquito rearing}

After collection from the field, fourth-instar larvae and pupae were transported directly to the insectary at Oxford University Clinic Research Unit, District 5, HCMC, where they completed development in the same water in which they were collected (with no additional food provided). After emergence, the field-reared mosquitoes were maintained under controlled environmental conditions as follows: $28{ }^{\circ} \mathrm{C}$; $65-85 \%$ relative humidity; and a 12:12 h light:dark cycle with sugar and water $a d$ libitum. All mosquitoes were confirmed as either $A e$. aegypti or Ae. albopictus visually, based on morphological characteristics. Aedes aegypti mosquitoes were further confirmed at a molecular level, using Ae. aegypti-specific primers targeting the RPS17 gene, in a multiplex RT-PCR that also targeted DENV (see Dengue virus diagnostics below). After emergence, up to 5 females and 5 males were maintained in individual cups, to allow mating. For maintenance, mosquitoes were allowed access to water and sucrose solution ad libitum until one day before blood-feeding (irrespective of the source of blood), when water and sugar were then removed to starve the females. The same rearing conditions were used for the colonised mosquitoes in the second set of experiments.

\section{Infectious and non-infectious blood meals}

$\mathrm{Up}$ to $20 \mathrm{~F}_{0}$ mosquitoes were maintained in cups (1:1 ratio of females:males) and offered the blood of NS1positive dengue patients via artificial membrane feeders. Venous blood, drawn into an EDTA tube, was collected from patients for viral quantification, serotyping and to be offered to mosquitoes within $1 \mathrm{~h}$ of the blood draw. After
30 min of access to the viremic blood, females were sorted and only fully engorged were retained. All mosquitoes were kept in cups under controlled conditions, as described above, until the non-infectious blood meal was offered to the surviving females. Blood from healthy donors was drawn and provided to the $\mathrm{F}_{0}$ females on Day 14 or Day 10 , according to the experiment set. Only fully engorged females were retained and placed into individual cups containing wet cotton balls for oviposition.

\section{Hatching and harvesting $F_{1}$ mosquitoes}

Five to 7 days after the second blood-feeding, eggs from each $\mathrm{F}_{0}$ female were hatched in cups and kept in incubators until mosquito emergence, for both sets of experiments. For the first experiment with Ae. aegypti and Ae. albopictus, the whole body of males from $\mathrm{F}_{0}$ DENV-positive females were collected for DENV screening 14 days after mosquito emergence. For $F_{1}$ females, saliva was collected for inoculation into five naïve mosquitoes, as previously described [21], before their whole bodies were collected for DENV screening. Inoculated mosquitoes were then harvested 7 days post-injection and screened for DENV in pools, according to the saliva source. For the second set of experiments, the $F_{1}$ progeny was collected on Day 7 after emergence and whole bodies were screened for DENV in pools of up to 8 individuals, with males and females tested separately. DENV detection was performed as described in the following section.

\section{Dengue virus diagnostics}

DENV plasma viremia levels in patient blood samples were measured by a validated, quantitative serotype-specific RT-PCR assay [22]. Only fully engorged females that laid eggs after the non-infectious blood meal were screened for DENV infection. Briefly, mosquitoes were homogenised with a $2 \mathrm{~mm}$ glass bead in squash buffer $(10 \mathrm{mM}$ Tris, $1 \mathrm{mM}$ EDTA, $50 \mathrm{mM} \mathrm{NaCl}[\mathrm{pH} 8.2]$ and proteinase $\mathrm{K})$, followed by an incubation period of $56^{\circ} \mathrm{C}$ for $10 \mathrm{~min}$, $98{ }^{\circ} \mathrm{C}$ for $15 \mathrm{~min}$ and a cool down step to $15^{\circ} \mathrm{C}$. Each sample was tested in a duplex RT-PCR, designed to amplify a conserved 3'-UTR region of all four DENV serotypes, and the Rps17 gene from Ae. aegypti as an internal control, as previously described [23], using a LightCycler480 Instrument (Roche, Mannheim, Germany) with the following run conditions: $50{ }^{\circ} \mathrm{C}$ for $15 \mathrm{~min}, 95^{\circ} \mathrm{C}$ for $2 \mathrm{~min}$, followed by 45 amplification cycles of $95^{\circ} \mathrm{C}$ for $15 \mathrm{~s}, 60^{\circ} \mathrm{C}$ for $30 \mathrm{~s}$ and a final cooling step of $40^{\circ} \mathrm{C}$ for $10 \mathrm{~s}$.

\section{Calculations of vertical transmission}

We estimated three related indices of vertical transmission, to help understand different contributions that 


\section{(See figure on next page.)}

Fig. 1 Flowchart of patient enrolment and mosquito processing to compare vertical transmission frequencies between field-reared Ae. aegypti and Ae. albopictus mosquitoes. The flowchart depicts the fate of mosquitoes as they were processed, in order to determine the frequency of vertical transmission of $\mathrm{F}_{0}$ females after feeding on blood from acutely-infected dengue patients admitted to the Hospital of Tropical Diseases (HTD) in Ho Chi Minh City, Vietnam. Boxes in red represent the samples excluded from analysis

each has to transmission dynamics. First, we calculated the number of $\mathrm{F}_{0}$ mothers who are capable of transmitting virus to any number of their progeny. Secondly, we calculated the proportion of their progeny, to whom they successfully transmit that virus. And finally, we calculated the proportion of infected progeny that are capable of expectorating virus in their saliva, a necessary element for re-introducing virus back into the horizontal transmission cycle.

We calculated these indices in two different ways, based on different denominators. The first formulas may be interpreted as providing a more refined estimate of vertical transmission, as it is based on a restricted subset of $\mathrm{F}_{0}$ mosquitoes with a confirmed DENV infection only, including all eggs derived from those females. The second, more inclusive estimate encapsulates all females who fed upon the infectious blood meal and laid eggs, irrespective of the eventual infectious status of the $\mathrm{F}_{0}$ female. This estimate is more conservative and is expected to more closely represent estimates derived from field-based studies. The precise formulas used to calculate these indices are listed below. The numbers in brackets at the end of the equation represents the number of mosquitoes used as the denominator in each equation. The bold text represents the equation (and denominator) used for the more inclusive, and thus conservative estimates of vertical transmission. All values can be found in the correspond flowchart (Fig. 1).

\section{Results}

Patient enrolment

We enrolled a total of 40 NS1-positive patients. RT-PCR was performed retrospectively to determine the viremia and virus serotype. Of the 30 patients' blood samples in whom we could confirm virus infection by RT-PCR, the predominant serotype was DENV-2 (50\%) with the range of viremia of $5.70-7.51 \log _{10}$ copies $/ \mathrm{ml}$. The second most predominant serotype was DENV-1 (30\%) with viremia of 5.31-7.97 $\log _{10}$ copies/ml and lastly was DENV-4 (20\%) with viremia of 6.67-8.32 $\log _{10}$ copies/ml. Characteristics of the enrolled patients are found in Additional file 1: Table S1. Ten patient blood samples were excluded from the study because they were DENV-negative when tested by RT-PCR.

\section{Species composition of field-reared mosquitoes}

Between November 2018 and September 2019, we collected a total of 3720 Aedes larvae/pupae over a period of 38 weeks, from up to 4 collection sites in District 8 , HCMC, each week. The predominant species was $A e$. aegypti with 3528 (94.8\% of total) individuals collected (1802 females and 1726 males). On average, $47 \mathrm{Ae}$. aegypti female mosquitoes were available for experiments on a weekly basis. We also collected 192 Ae. albopictus mosquitoes $(5.16 \%$ of total), with 79 females and 114 males. The majority of remaining individuals collected were culicine mosquitoes.

\section{Frequency of mothers that transmit virus to any of their progeny}

$$
=\frac{\text { Number of DENV infected mothers with at least one DENV positive progeny }}{\text { Total number of DENV infected ( }+ \text { uninfected) mothers that laid eggs after second blood meal }(41 / \mathbf{8 4})}
$$

$$
\begin{aligned}
& \text { Proportion of F1 progeny that inherited virus from infected mothers } \\
& =\frac{\text { Number of surviving F1progeny that were infected with virus }}{\text { Total number of eggs laid by all DENV infected }(+ \text { uninfected }) \text { mothers }(911 / 2533)}
\end{aligned}
$$

$$
\begin{aligned}
& \text { Proportion of infected female progeny capable of transmitting virus horizontally } \\
& =\frac{\text { Number of DENV infected female progeny with infectious DENV in their saliva }}{\text { Total number of eggs laid by all DENV infected (+uninfected)mothers }(911 / \mathbf{2 5 3 3})}
\end{aligned}
$$




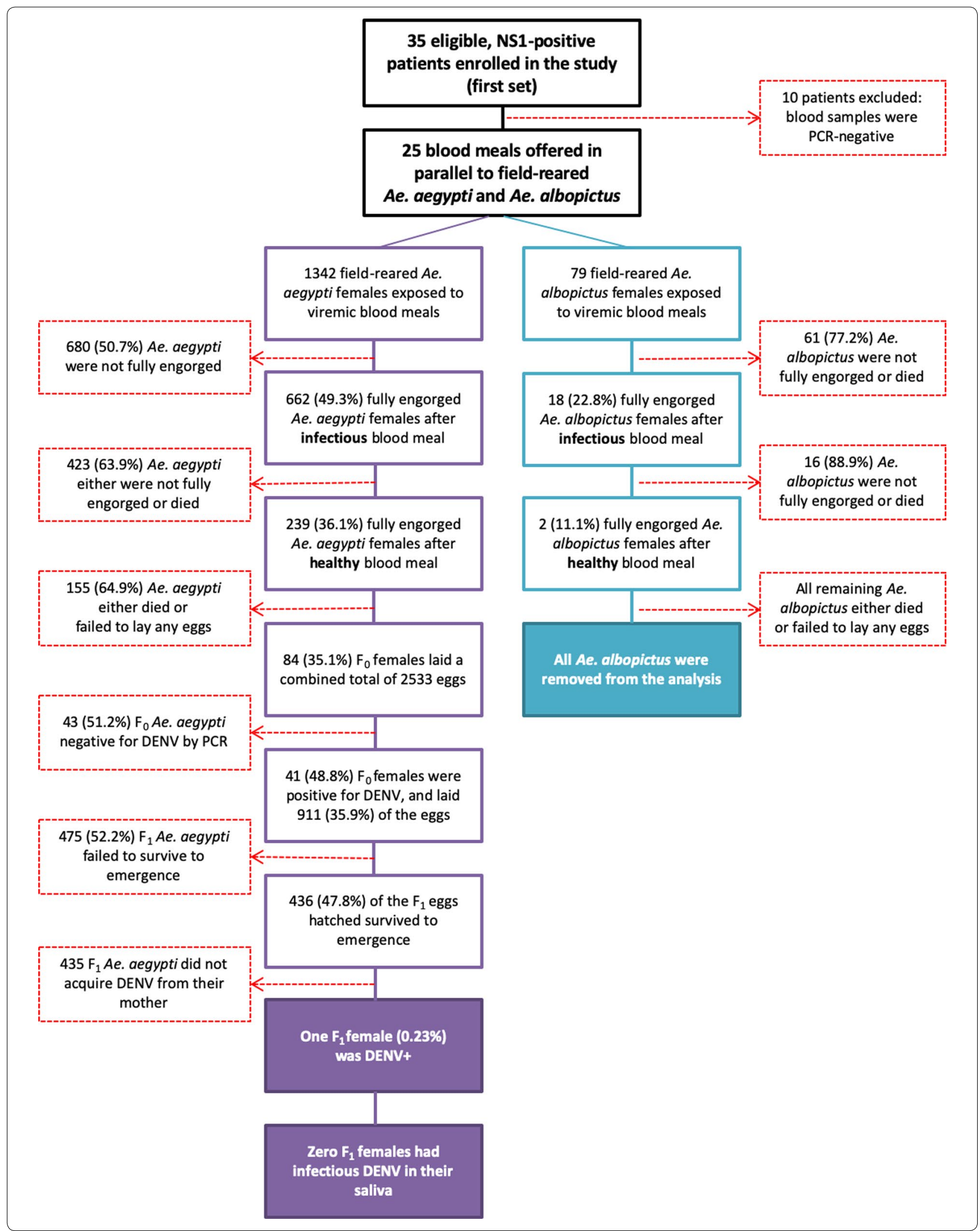




\section{Estimates of vertical transmission in field-reared $A e$. aegypti and Ae. albopictus}

We examined vertical DENV transmission by fieldreared Ae. aegypti and Ae. albopictus after they had fed on viremic blood meals. The 25 independent viremic blood meals resulted in 662 of $1342 \mathrm{~F}_{0}$ Ae. aegypti females $(49.3 \%$ of the total) being engorged. Approximately one third of these females $(n=239)$ fed upon the non-infectious blood meal, administered on Day 14, which stimulated the second gonotrophic cycle. Of these $239 \mathrm{~F}_{0}$ females, 84 survived long enough to lay a total of 2533 eggs. Retrospectively, we determined that only 41 of these 84 females were infected with DENV. The eggs $(n=911)$ from these 41 DENV infected $F_{0}$ 's developed into 436 mature adults. All $214 \mathrm{~F}_{1}$ males were negative for DENV infection. Only one (CI: 0.00-0.03) of the $222 \mathrm{~F}_{1}$ females tested positive for DENV in her body; the latter was infected with DENV-2 serotype and had a Cq value of 33.11. None of the saliva samples collected from any $F_{1}$ female contained infectious virus that amplified in the in vitro transmission assay (Fig. 1).

Thus, our results suggest that vertical transmission under conditions similar to the natural history is rare; for every 41 DENV-infected, field-reared females that survive and reproduce after 14 days, only 1 (2.4\%) would transmit virus to the progeny. If we calculate based on the $\mathrm{F}_{1}$ progeny that emerged to adults $(n=436)$, the percentage of DENV-infected progeny from a DENV-infected mother drops to $0.23 \%$ (Fig. 1 ). Using a more conservative denominator, which includes all females that fed upon an infectious blood meal and laid eggs, regardless their infection status, this represents 1 female who transmits DENV to her progeny for every 84 females (1.2\%). With only 1 of all the 911 eggs laid by an infected mother having acquired virus, we calculate that $0.11 \%$ of progeny born to a DENV-infected mother in the second gonotrophic cycle would carry DENV (Fig. 2).

For Ae. albopictus, 18 of 79 females (23.0\%) who fed on the infectious blood meal survived and fed upon the second, healthy blood meal. However, only 2 of the 18 females $(11.1 \%)$ become engorged, and neither produced a second gonotrophic cycle. As such, we were unable to assess the frequency of vertical transmission of DENV in Ae. albopictus (Fig. 1).

\section{Vertical transmission frequencies of field-reared $v s$ laboratory-reared Ae. aegypti females}

In this second set of experiments, we exposed an additional 460 field-reared Ae. aegypti females, alongside 946 laboratory-reared Ae. aegypti females, to 5 patientderived blood meals. We tested pools of female progeny, rather than individual mosquitoes, for the presence of DENV infection.
At Day 10 post-virus exposure, the surviving 222 engorged field-reared females were exposed to healthy blood meals, of which 67 females (30.2\%) fed to repletion. Eighteen females were positive for DENV and laid $879 \mathrm{~F}_{1}$ eggs in total. A total of $558 \mathrm{~F}_{1}$ mosquitoes were tested in 108 pools for DENV infection and all tested negative. For laboratory-reared females, there were 571 engorged individuals after the first infectious blood meal and 205 after the second one. In total, 72 females were both infected with DENV and laid eggs, from which 3240 mature $F_{1}$ mosquitoes were reared. All 564 pools for screening were again test-negative for DENV (Additional file 2: Figure S1).

\section{Discussion}

Our data show that less than 3\% of field-reared females who are known to have fed upon a patient-derived viremic blood meal, are capable of transmitting DENV to any of their progeny two weeks later. We further demonstrate that fewer than one quarter of a percent of mosquitoes born to these virus-exposed mothers will acquire infection. If we calculate the frequency using all eggs laid by females both infected and uninfected that were exposed to the same blood meals, this number goes to less than 1 of 2500 eggs laid. Finally, even less are likely to re-introduce virus into the human population.

The role of vertical virus transmission has been considered under multiple scenarios. It has been stimulated by theory that transmission of virus through the germ line may provide an alternate mechanism for virus maintenance in nature. Some studies suggest that vertical transmission allows re-emergence and maintenance of arboviruses in a vector population in different scenarios: (i) in between non-epidemic periods; (ii) when the density of mosquito population is low (due to winter diapause); or (iii) in the absence of any viremic hosts [24, 25]. It has also been suggested that virus in diapausing eggs can remain viable until mosquito emergence, thus the virus can multiply and be re-introduced horizontally to the vertebrate host population $[26,27]$.

While vertical virus transmission in the field has been documented since it was first described in 1906 [28-30], there is insufficient evidence to demonstrate it contributes to re-emergence or maintenance of viruses in nature. A recent study described DENV vertical transmission in field-collected immature mosquitoes by detecting the virus in $0.4 \%$ of the pooled samples, and even this low VT frequency could explain the persistence of the virus during interepidemic times in Africa [31]. However, those studies have failed to test the important hypothesis, that vertically acquired virus can actually be horizontally transmitted [8]. 


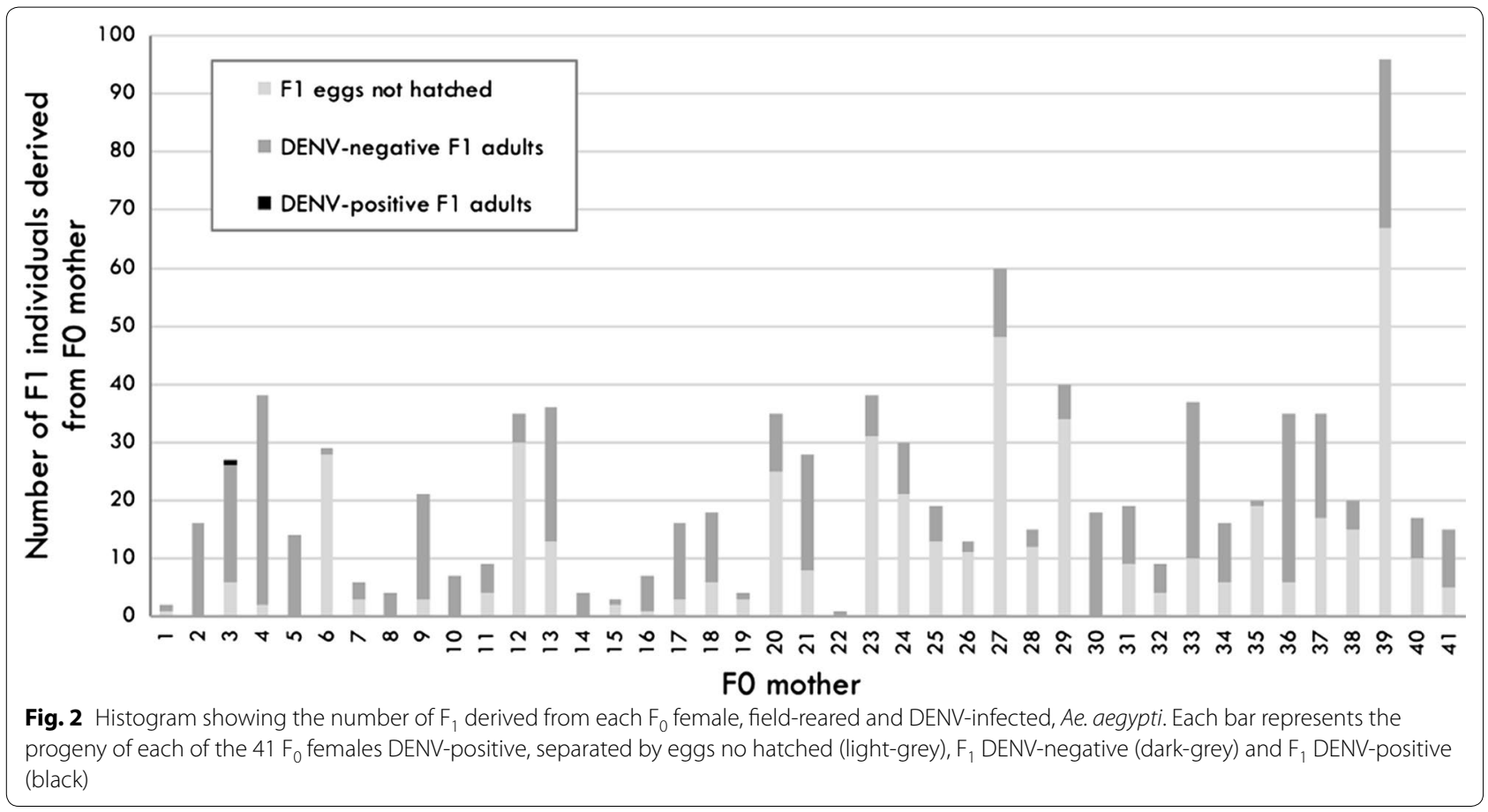

Mimicking natural transmission dynamics as closely as possible and using field-reared mosquitoes exposed to blood drawn from acutely viremic dengue patients, our data support the premise that vertical transmission is a relatively rare occurrence $[8,31,32]$. Moreover, we could not detect virus in the saliva of our single, vertically-infected female. Our mosquito sample size was relatively small, but based on our data, it suggests that re-introduction of the virus to the human population via horizontal transmission is an uncommon event. Alternative mechanisms to explain re-emergence of virus within a human population may be introduction of virus from another location, or continued asymptomatic carriage in the human population.

A stable and systemic dissemination of viruses acquired by oral feeding in Aedes mosquitoes is important as a determinant for VT to subsequent progeny [33, 34]. This dissemination can take days or weeks to occur $[35,36]$ and thus most vertical transmission must occur in the female later gonotrophic cycles [32, 33, 37]. Before stimulating a second gonotrophic cycle with a healthy blood meal, we allowed 14 days for virus replication, a period sufficiently long to achieve systemic viral dissemination, and high viral titres, in our infection model [21, 38, 39]. Despite this, the proportion of DENV-infected progeny we detected was still below $0.5 \%$. Future experiments should consider stimulation of more frequent and additional gonotrophic cycles, to promote cell proliferation and associated viral replication. This may assist detection of an increased prevalence of vertical transmission of virus from a DENV-infected mother to her progeny.

Previous studies have reported notably high prevalence of vertical transmission and suggest a significant contribution of this phenomenon to the maintenance of DENV in nature. One recently published estimate of VT ranges from 14.8 to $23.5 \%$ in Ae. aegypti collected in ovitraps [14]. Yet these values are derived from calculations of the number of positive ovitraps, rather than the number of positive individuals contained within that trap. With an experimental design more similar to our own study, Sánchez-Vargas et al. [34] reported virus infection frequencies varying from 55 to $68.6 \%$ in $\mathrm{F}_{1}$ mosquitoes derived from infected mothers. They did however use a blood meal spiked with DENV-2 to first infect mosquitoes, and tested $F_{1}$ mosquitoes in pools, rather than individually [34]. Pooling of samples can lead to an overestimation of the frequency of occurrence of VT and reduces the sensitivity of DENV-detection in those samples [8].

A limiting factor in achieving a large sample size in this study was our restricted criteria for 'field-reared' mosquitoes by selecting only fourth-instars and larvae, as we intended to maximise the duration of their development under field conditions. We also observed relatively high mortality after the second blood meal, prior to $\mathrm{F}_{0}$ females laying eggs, for the field-reared mosquitoes. One possible reason for this is the females' age, in combination with variable and suboptimal rearing conditions during 
the larval stage in the field. Such suboptimal conditions may include nutritional stress, over-/under-crowding and fluctuating temperatures which can influence fitness and longevity [39-41], further highlighting that vertical transmission in the field is likely an uncommon event. As such, while our sample sizes are small, we are confident that the experimental design and resulting data are representative of realistic conditions.

\section{Conclusions}

To the best of our knowledge, this is the first study to assess vertical transmission of DENV using fieldreared Ae. aegypti mosquitoes exposed to viremic blood from patients. Our data demonstrate infrequent transmission of virus from mother to progeny and can suggest that vertically acquired virus may not play a substantial role in dengue virus transmission vertically.

\section{Supplementary information}

Supplementary information accompanies this paper at https://doi. org/10.1186/s13071-020-04334-5.

Additional file 1: Table S1. Characteristics of the 40 dengue patients enrolled in the study.

Additional file 2: Figure S1. Flowchart of patient enrolment and mosquito processing to compare vertical transmission frequencies between field- and laboratory-reared Ae. aegypti mosquitoes. The flowchart depicts the fate of mosquitoes as they were processed, in order to determine the frequency of vertical transmission of $F_{0}$ females after feeding on blood from acutely-infected dengue patients admitted to the Hospital of Tropical Diseases (HTD) in Ho Chi Minh City, Vietnam. Boxes in red represent the samples excluded from analysis.

\section{Abbreviations}

VT: vertical transmission; HT: horizontal transmission; HTD: Hospital for Tropical Diseases; EC: Ethics Committee; NS1: non-structural protein 1; HCMC: Ho Chi Minh City; RT-PCR: reverse transcriptase polymerase chain reaction; 3'UTR: 3 prime untranslated region.

\section{Acknowledgements}

We would like to thank the patients enrolled in this study, the clinicians and nursing staff in Ward D at the Hospital for Tropical Diseases for their support.

\section{Authors' contributions}

LBC, BW, NTP and CPS participated to the study design. NTVT, DSG and LBC coordinated patient enrolment and sample collection. DTVK, TTV, VTN, NTG, HTTV, HTXT, LTD, VTL, HLAH and NTVT performed the experiments. LBC, DSG and TTV coordinated the data management. LBC and DSG analysed the data. CPS contributed with funding and reagents. LBC, DSG and CPS wrote the manuscript. All authors read and approved the final manuscript.

\section{Funding}

This study was supported by a Wellcome trust strategic award.

\section{Availability of data and materials}

All relevant data generated or analysed during this study are included in this published article and its additional files.

\section{Ethics approval and consent to participate}

All work was conducted at the Hospital for Tropical Diseases (HTD), Ho Chi Minh City (HCMC), Vietnam. The enrolment of patients for provision of viremic blood meals to WT Ae. aegypti and Ae. albopictus was approved by the Ethics Committee HTD EC and OxTREC (approval nos. HTD EC CS/NĐ/14/12 and OxTREC 45-14). Informed consent was obtained from all participants by trained study staff.

\section{Consent for publication}

Not applicable.

\section{Competing interests}

The authors declare that they have no competing interests.

\section{Author details}

1 Oxford University Clinical Research Unit, Wellcome Trust Major Overseas Programme, District 5, Ho Chi Minh City, Vietnam. ${ }^{2}$ Hospital for Tropical Diseases, District 5, Ho Chi Minh City, Vietnam. ${ }^{3}$ Institute for Vector Borne Disease, Monash University, Clayton, Melbourne, VIC 3168, Australia.

Received: 8 July 2020 Accepted: 1 September 2020

Published online: 14 September 2020

\section{References}

1. Brady OJ, Gething PW, Bhatt S, Messina JP, Brownstein JS, Hoen AG, et al. Refining the global spatial limits of dengue virus transmission by evidence-based consensus. PLoS Negl Trop Dis. 2012;6:e1760.

2. Bhatt $\mathrm{S}$, Gething PW, Brady OJ, Messina JP, Farlow AW, Moyes CL, et al. The global distribution and burden of dengue. Nature. 2013;496:504-7.

3. Lwande OW, Obanda V, Lindström A, AhIm C, Evander M, Näslund J, et al. Globe-trotting Aedes aegypti and Aedes albopictus: risk factors for arbovirus pandemics. Vector Borne Zoonotic Dis. 2019;20:71-81.

4. Kamal M, Kenawy MA, Rady MH, Khaled AS, Samy AM. Mapping the global potential distributions of two arboviral vectors Aedes aegypti and Ae. albopictus under changing climate. PLoS ONE. 2018;13:e0210122.

5. Christophers S. Its life history, bionomics and structure. Aedes aegypti the yellow fever mosquito. London: Cambridge University Press; 1960.

6. Tesh RB, Shroyer DA. The mechanism of arbovirus transovarial transmission in mosquitoes: San Angelo virus in Aedes albopictus. Am J Trop Med Hyg. 1980;29:1394-404.

7. Rosen L. Mechanism of vertical transmission of the dengue virus in mosquitoes. C R Acad Sci. 1987;3:347-50

8. Lequime S, Lambrechts L. Vertical transmission of arboviruses in mosquitoes: a historical perspective. Infect Genet Evol. 2014;28:681-90.

9. Joshi V, Mourya DT, Sharma RC. Persistence of dengue-3 virus through transovarial transmission passage in successive generations of Aedes aegypti mosquitoes. Am J Trop Med Hyg. 2002;67:158-61.

10. Farnesi LC, Martins AJ, Valle D, Rezende GL. Embryonic development of Aedes aegypti (Diptera: Culicidae): influence of different constant temperatures. Mem Inst Oswaldo Cruz. 2009;104:124-6.

11. Hardy JL, Rosen L, Kramer LD, Presser SB, Shroyer DA, Turell MJ. Effect of rearing temperature on transovarial transmission of St. Louis encephalitis virus in mosquitoes. Am J Trop Med Hyg. 1980;29:963-8.

12. Mitchell CJ, Miller BR. Vertical transmission of dengue viruses by strains of Aedes albopictus recently introduced into Brazil. J Am Mosq Control Assoc. 1990;6:251-3.

13. Kramer LD, Ebel GD. Dynamics of flavivirus infection in mosquitoes. Adv Virus Res. 2003:60:187-232.

14. Satoto TBT, Listyantanto A, Agustjahjani SD, Josef HK, Widartono BS. Vertical transmission of dengue virus in the Yogyakarta airport area. Environ Health Prev Med. 2018;23:22.

15. Hartanti MD, Suryani, Tirtadjaja IA. Dengue virus transovarial transmission by Aedes aegypti. Universa Med. 2010;29:65-70.

16. Fine PEM. Vectors and vertical transmission: an epidemiologic perspective. Ann N Y Acad Sci. 1975;266:173-94.

17. Watts DM, Harrison BA, Pantuwatana S, Klein TA, Burke DS. Failure to detect natural transovarial transmission of dengue viruses by Aedes aegypti and Aedes albopictus (Diptera: Culicidae). J Med Entomol. $1985: 22: 261-5$ 
18. Anders KL, Nga LH, Van Thuy NT, Van NT, Tam CT, Tai LTH, et al. Households as foci for dengue transmission in highly urban Vietnam. PLoS Negl Trop Dis. 2015;9:e0003528.

19. Ilkal MA, Dhanda V, Hassan MM, Mavale M, Mahadev PV, Shetty PS, et al. Entomological investigations during outbreaks of dengue fever in certain villages in Maharashtra state. Indian J Med Res. 1991;93:174-8.

20. Ross PA, Endersby-Harshman NM, Hoffmann AA. A comprehensive assessment of inbreeding and laboratory adaptation in Aedes aegypti mosquitoes. Evol Appl. 2019;12:572-86.

21. Nguyen NM, Kien DTH, Tuan TV, Quyen NTH, Tran CNB, Thi LV, et al. Host and viral features of human dengue cases shape the population of infected and infectious Aedes aegypti mosquitoes. Proc Natl Acad Sci USA. 2013;110:9072-7.

22. Hue KDT, Tuan TV, Thi HTN, Bich CTN, Le Anh HH, Wills BA, et al. Validation of an internally controlled one-step real-time multiplex RT-PCR assay for the detection and quantitation of dengue virus RNA in plasma. J Virol Methods. 2011;177:168-73.

23. Frentiu FD, Zakir T, Walker T, Popovici J, Pyke AT, van den Hurk A, et al. Limited dengue virus replication in field-collected Aedes aegypti mosquitoes infected with Wolbachia. PLoS Negl Trop Dis. 2014:8:e2688.

24. Leake CJ. Transovarial transmission of arboviruses by mosquitoes. New York: Academic Press; 1984.

25. Angel B, Joshi V. Distribution and seasonality of vertically transmitted dengue viruses in Aedes mosquitoes in arid and semi-arid areas of Rajasthan, India. J Vector Borne Dis. 2008:45:56-9.

26. Reeves W. Overwintering of arboviruses. Prog Med Virol. 1974;17:193-220

27. Guo X, Zhao T, Dong Y, Lu B. Survival and replication of dengue-2 virus in diapausing eggs of Aedes albopictus (Diptera: Culicidae). J Med Entomol. 2007:44:492-7.

28. Marchoux É, Simond PL. Etudes sur la fièvre jaune - Deuxième mémoire. Ann Inst Pasteur. 1906;20:1-25.

29. Kuno G, Chang GJJ. Biological transmission of arboviruses: reexamination of and new insights into components, mechanisms, and unique traits as well as their evolutionary trends. Clin Microbiol Rev. 2005;18:608-37.

30. Ferreira-De-Lima VH, Lima-Camara TN. Natural vertical transmission of dengue virus in Aedes aegypti and Aedes albopictus: a systematic review. Parasites Vectors. 2018;11:77

31. Heath CJ, Grossi-Soyster EN, Ndenga BA, Mutuku FM, Sahoo MK, Ngugi $\mathrm{HN}$, et al. Evidence of transovarial transmission of Chikungunya and dengue viruses in field-caught mosquitoes in Kenya. PLoS Negl Trop Dis. 2020;14:e0008362.
32. Danis-Lozano R, Díaz-González EE, Malo-García IR, Rodríguez MH, RamosCastañeda J, Juárez-Palma L, et al. Vertical transmission of dengue virus in Aedes aegypti and its role in the epidemiological persistence of dengue in central and southern Mexico. Trop Med Int Health. 2019;24:1311-9.

33. Tesh RB. Transovarial transmission of arboviruses in their invertebrate vectors: Current topics in vector research, vol. 2. New York: Praeger Scientific; 1984. p. 57-76

34. Sánchez-Vargas I, Harrington LC, Doty JB, Black WC, Olson KE. Demonstration of efficient vertical and venereal transmission of dengue virus type-2 in a genetically diverse laboratory strain of Aedes aegypti. PLoS Negl Trop Dis. 2018;12:e0006754.

35. Salazar MI, Richardson JH, Sánchez-Vargas I, Olson KE, Beaty BJ. Dengue virus type 2: replication and tropisms in orally infected Aedes aegypti mosquitoes. BMC Microbiol. 2007;7:9.

36. Moore PR, Johnson PH, Smith GA, Ritchie SA, van den Hurk AF. Infection and dissemination of dengue virus type-2 in Aedes aegypti, Aedes albopictus, and Aedes scutellaris from the Torres Strait, Australia. J Am Mosq Control Assoc. 2007;23:383-8.

37. Shroyer DA. Vertical maintenance of dengue-1 virus in sequential generations of Aedes albopictus. J Am Mosq Control Assoc. 1990;6:312-4.

38. Whitehorn J, Kien DTH, Nguyen NM, Nguyen HL, Kyrylos PP, Carrington LB, et al. Comparative susceptibility of Aedes albopictus and Aedes aegypti to dengue virus infection after feeding on blood of viremic humans: implications for public health. J Infect Dis. 2015;212:1182-90.

39. Carrington LB, Tran BCN, Le NTH, Luong TTH, Nguyen TT, Nguyen PT, et al. Field- and clinically derived estimates of Wolbachia-mediated blocking of dengue virus transmission potential in Aedes aegypti mosquitoes. Proc Natl Acad Sci USA. 2018;115:361-6.

40. Couret J, Dotson E, Benedict MQ. Temperature, larval diet, and density effects on development rate and survival of Aedes aegypti (Diptera: Culicidae). PLoS ONE. 2014:9:e87468.

41. Gunathilaka N, Upulika H, Udayanga L, Amarasinghe D. Effect of larval nutritional regimes on morphometry and vectorial capacity of Aedes aegypti for dengue transmission. Biomed Res Int. 2019;2019:1-11.

\section{Publisher's Note}

Springer Nature remains neutral with regard to jurisdictional claims in published maps and institutional affiliations.
Ready to submit your research? Choose BMC and benefit from:

- fast, convenient online submission

- thorough peer review by experienced researchers in your field

- rapid publication on acceptance

- support for research data, including large and complex data types

- gold Open Access which fosters wider collaboration and increased citations

- maximum visibility for your research: over $100 \mathrm{M}$ website views per year

At BMC, research is always in progress.

Learn more biomedcentral.com/submissions 